\title{
Late mortality after severe traumatic brain injury in New South Wales: a multicentre study
}

\section{Ian J Baguley MB BS, FAFRM, PhD, Rehabilitation Specialist $^{\dagger}$ \\ Melissa T Nott BAppSc(Hons), PhD Clinical Research Officer ${ }^{1}$ \\ Alison A Howle BSpPath, Researcher' \\ Grahame K Simpson BSocStud, MA, PhD, Research Team Leader/ Senior Social Worker ${ }^{2}$ \\ Stuart Browne MB BS, MD, FAFRM, Staff Specialist ${ }^{3}$ \\ A Clayton King MB BS, MD, FAFRM Medical Director \\ Rachel E Cotter BA(Hons) Research Assistant \\ Adeline Hodgkinson MB BS, FAFRM Director ${ }^{2}$ \\ 1 Brain Injury Rehabilitation Service, Westmead Hospital, Sydney, NSW. \\ 2 Brain Injury Rehabilitation Unit, Liverpool Hospital, Sydney, NSW. \\ 3 Brain Injury Rehabilitation Service Royal Rehabilitation Centre Sydney, \\ Sydney, NSW. \\ lan.Baguley@ swahs.health.nsw.gov.au}

MJA 2012; 196: 40-45 doi: 10.5694/mjall.10090

Editorial p 9 evere traumatic brain injury (TBI), a leading cause of death and disability worldwide, ${ }^{1}$ is a particularly important societal issue because of its high economic and personal cost. ${ }^{2}$ Australian studies have found acute mortality rates of $30 \%-$ $35 \%$ during the first 6 months after severe TBI. ${ }^{3-5}$ Recent Australian estimates on the lifelong economic cost of moderate-to-severe TBI assumed that "patients surviving year 1 [postinjury] reverted to the mortality risk for the general population". ${ }^{6}$ However, further data are required to determine the accuracy of this assumption and to improve the robustness of future modelling.

The only Australian investigation examining late (ie, greater than 1 year) all-cause mortality for people with severe TBI involved a singlecentre cohort study of 476 patients undergoing rehabilitation in New South Wales. ${ }^{7}$ The standardised mortality ratio (SMR) - the ratio of observed mortality in the study group compared with that expected of an age- and sex-matched population after discharge indicated a fourfold increase in mortality; this ratio is higher than international long-term mortality estimates, which range from 1.1 to $3.1 .^{3,8-14}$

An increased understanding of risk factors associated with long-term mortality is important for informing clinical management. Current knowledge, predominantly from overseas studies, has identified increasing age, ${ }^{1,7,10,15-17}$ male sex ${ }^{10,17}$ a history of psychiatric illness, ${ }^{7,8,11}$ alcohol and drug misuse, ${ }^{11}$ epilepsy, $9,13,18$ and functional dependence ${ }^{12,13,17}$ as important variables. Studies suggest that post-TBI death rates are equivalent to those of the general population for some diseases (eg, neoplasia),

Abstract

Objectives: To determine the long-term mortality pattern of adults with severe traumatic brain injury (TBI), and to identify the risk factors associated with death in this group.

Design, patients and setting: Inception cohort study of 2545 adults consecutively discharged from one of three metropolitan tertiary, post-acute inpatient rehabilitation services of the New South Wales Brain Injury Rehabilitation Program from 1 January 1990 to 1 October 2007 after inpatient rehabilitation for primary $\mathrm{TBI}$.

Main outcome measure: Survival status at 1 October 2009.

Results: 258 deaths were recorded in this sample, yielding a standardised mortality ratio of $3.19(95 \% \mathrm{Cl}, 2.80-3.60)$. Risk of death remained elevated above societal norms for at least 8 years after discharge from rehabilitation. Mortality risk was increased by: functional dependence at discharge; age at injury; pre-injury drug and alcohol misuse; pre-injury epilepsy; and discharge to an aged care facility. The risk of death from external causes, and respiratory system and nervous system disorders was six to seven times higher, and the risk of death from disorders of the digestive system, and mental and behavioural disorders was five times higher in adults with severe TBI than in the general population.

Conclusions: People who survive to discharge from inpatient rehabilitation following a severe TBI were found to have a sustained increase in risk of death for eight years post discharge. Various demographic and injury-related variables selectively increase mortality risk and may be modifiable in order to reduce the observed increase in mortality.

while mortality rates from other causes (eg, respiratory illnesses, aspiration pneumonia) are significantly elevated.

Here we further investigate these issues in an Australian data linkage study of people with severe TBI who were discharged from the three adult inpatient units of the NSW Brain Injury Rehabilitation Program (BIRP) in metropolitan Sydney. The study aimed to: (i) determine the long-term all-cause mortality pattern for this inception cohort; (ii) identify associated risk factors; and (iii) examine mortality rates for specific causes of death.

\section{Methods}

After obtaining approval from the appropriate local institutional ethics committees, we searched databases and medical records to identify consecutive rehabilitation admissions of patients with TBI since the NSW BIRP commenced on 1 January $1990 .^{19,20}$ The resultant inception cohort was derived from this integrated, statewide, specialist inpatient and community-based rehabilitation program for people who had sustained a severe TBI. Admissions were screened against the following inclusion criteria: age 16-70 years at time of injury; primary BIRP admission; severe TBI (Glasgow Coma Scale score $<9$ and/ or duration of post-traumatic amnesia $>1$ day $\left.^{21}\right)$; and discharged alive before 1 October 2007. This date was selected to coincide with introduction of the Lifetime Care and Support Scheme for adults severely injured in a motor vehicle accident in NSW. Admissions for subsequent brain injuries for any patient were excluded, 
1 Age distribution, sex and pre-injury medical history of the 2545 patients with severe traumatic brain injury, and results of the univariate Cox regression analysis

\begin{tabular}{|c|c|c|c|c|}
\hline Variable & No. of patients & Measure & Hazard ratio $(95 \% \mathrm{Cl})$ & $P$ \\
\hline \multicolumn{5}{|l|}{ Demographic characteristics } \\
\hline Mean age* ${ }^{\dagger}(\mathrm{SD})$ & 2545 & $35(14)$ & & \\
\hline 16-20 years (reference group for Cox regression) & & $374(15 \%)$ & 1 & \\
\hline $21-25$ years & & $459(18 \%)$ & $0.65(0.32-1.32)$ & 0.24 \\
\hline $26-35$ years & & $591(23 \%)$ & $1.44(0.81-2.55)$ & 0.21 \\
\hline $36-45$ years & & $457(18 \%)$ & $2.82(1.64-4.86)$ & $<0.001$ \\
\hline$\geqslant 46$ years & & $664(26 \%)$ & $5.36(3.23-8.88)$ & $<0.001$ \\
\hline Sex* & 2545 & & & \\
\hline Female (reference group for Cox regression) & & $485(19 \%)$ & 1 & \\
\hline Male & & $2060(81 \%)$ & $2.39(1.57-3.64)$ & $<0.001$ \\
\hline \multicolumn{5}{|l|}{ Pre-injury medical history } \\
\hline Pre-injury history of traumatic brain injury* & 2545 & $61(2 \%)$ & $1.71(0.96-3.06)$ & 0.07 \\
\hline Pre-injury history of epilepsy* & 2545 & $77(3 \%)$ & $5.63(3.90-8.12)$ & $<0.001$ \\
\hline Pre-injury history of alcohol/drug misuse* & 2169 & & & \\
\hline History not reported in medical record & & $1533(71 \%)$ & 1 & \\
\hline History verified ${ }^{\ddagger}$ or reported in medical record & & $636(29 \%)$ & $3.01(2.34-3.87)$ & $<0.001$ \\
\hline Pre-injury history of psychiatric disorder* & 2168 & & & \\
\hline History not reported in medical record & & $1848(85 \%)$ & 1 & \\
\hline History verified or reported in medical record & & $320(15 \%)$ & $1.36(0.98-1.88)$ & 0.06 \\
\hline
\end{tabular}

as were secondary admissions for reviews and reassessments.

Data were collected on demographic, pre-injury, clinical, servicerelated and mortality variables (Box 1 , Box 2). Where available, the functional independence measure (FIM) ${ }^{22}$ was used to assess each patient's functional independence across 18 domains, with scores ranging from complete dependence (FIM score, 18) to complete independence (FIM score, 126). Patients' survival status was censored on 1 October 2009, providing a minimum 24-month interval between patient enrolment and the census date. The cohort list was provided to two national data registries - the National Death Index (NDI) and the National Coroners Information System (NCIS) - for matching, which yielded the date, cause of death and place of residence at time of death for deceased patients.

The NDI collates Australian death data from 1980, utilising Births, Deaths and Marriages information from each Australian state and territory and from the Australian Bureau of Statistics. The NDI records International classification of diseases (ICD) codes for cause of death extracted from death certificates, identifying primary and secondary causes of death. A probabilistic method matched our sample against NDI data using date of birth, family name, first name, and all derivatives of these. Guidelines for probabilistic matching and minimum thresholds for data acceptance recommended by the NDI were followed.

The NCIS collates Australian coronial information for people with external (eg, accidental or violent) causes of death from 1 July 2000. NCIS information assisted in determining intentionality for deaths from external causes after this date.

The primary cause of death stated on death certificates was coded using International classification of diseases, injuries and causes of death, 9th revision (ICD-9) for deaths from 1980 to 1996 and International statistical classification of diseases and related health problems, 10th revision (ICD-10) for deaths from 1997 onwards. Secondary causes of death or events associated with death were recorded as factors involved in the chain of events leading to the deaths of patients with TBI.

\section{Statistical analysis}

Descriptive statistics were calculated for all variables. Follow-up years (oth- erwise termed risk-exposure time) were calculated from date of discharge from rehabilitation to the census date or the date of death for those who had died. A population reference sample was constructed from Australian life-expectancy tables ${ }^{23}$ based on the age, sex and risk-exposure time for each person with TBI in the study. SMRs with $95 \% \mathrm{CIs}^{24}$ were calculated for all-cause deaths across the entire study period, for individual years after discharge and for individual causes of death. An SMR greater than 1.0 indicates an increased mortality risk compared with that of the matched reference population.

Cox proportional hazards regression analysis evaluated the effect of each potential risk factor on survival. Variables for which we had data completeness of $85 \%$ or greater were used in the univariate and multivariate analyses. Univariate results with $P$ values $<0.05$ were entered into the final multivariate regression. Hazard ratios with $95 \%$ CIs were calculated. A backwards stepwise method of regression was used to sequentially eliminate factors that did not independently contribute to risk of death.

Linear variables were grouped into ordinal categories for the purpose of 
2 Clinical, service and mortality variables of the 2545 patients with severe traumatic brain injury, and results of the univariate Cox regression analysis

Clinical variables

Brain injury cause

Motor vehicle accident-related

Fall/dive

Assault/non-accidental injury

$524(21 \%)$

Sport/recreation-related

$355(14 \%)$

Gunshot

$63(3 \%)$

$15(1 \%)$

Other traumatic brain injury

$65(3 \%)$

Mean functional independence measure scores (SD)*

Admission total score

Independent (108-126) (reference group for Cox regression)

Moderate assistance (55-107)

Maximal assistance (18-54)

Discharge total score

Independent (108-126) (reference group for Cox regression)

Moderate assistance (55-107)

Maximal assistance (18-54)

Occurrence of inhospital aspiration pneumonia*

Presence of percutaneous endoscopic gastrostomy during admission

Dysphagia reported/documented at discharge

Anticonvulsants prescribed at discharge

Guardianship order in place

Service variables

Median days from injury to rehabilitation admission (IQR)

Median length of stay for rehabilitation (IQR)*

$<30$ days (reference group for Cox regression)

31-60 days

$\geqslant 61$ days

Median days from injury to rehabilitation discharge (IQR)

Financial compensation for injury

Discharge destination*

Private house (reference group for Cox regression)

Ongoing rehabilitation/medical care

Care facility (nursing home or hostel)

\section{Mortality variables}

Deceased

Median years from discharge to death (IQR)

Place of residence at time of death

Private house

Hospital or care facility (nursing home/hostel)

$2144 \quad 69(37)$

$430(20)$

909 (42)

805 (38)

$2126 \quad 104(29)$

$1447(68)$

$466(22)$

$213(10)$

$219979(4 \%)$

$1692 \quad 311(18 \%)$

$1752 \quad 204(12 \%)$

$1467 \quad 465(32 \%)$

$2545 \quad 171(7 \%)$

$2534 \quad 25(24)$

$2545 \quad 37(66)$

1099 (43)

$546(22)$

900 (35)

$68(89)$

$\begin{array}{rc}2545 & 68(89) \\ 1851 & 826(45 \%)\end{array}$

2332

$1730(74 \%)$

$421(18 \%)$

$181(8 \%)$

1

$1.26(0.90-1.78) \quad 0.18$

$1.79(1.36-2.37) \quad<0.001$

$258(10 \%)$

$\begin{array}{rr}2545 & 258(10 \%) \\ 258 & 4.8(6.6)\end{array}$

253

$201(79 \%)$

$52(21 \%)$

$\mathrm{IQR}=$ interquartile range.

* Variables entered into the univariate Cox regression (sufficient available data).

Cox regression analysis. Discharge FIM scores were coded into three categories: maximal assistance (score 18-54, indicating a mean FIM item score of 3 or less); moderate assistance (score 55-107, indicating a mean FIM item score of 4 or 5); and independent (108-126, indicating a mean FIM item score of 6 or more). Age at injury was categorised by visual binning of equal percentiles on scanned cases $(20 \%$ of cases in each category): 16-20 years, 21-25 years, $26-35$ years, $36-45$ years, and $\geqslant 46$ years.

\section{Results}

The study sample of 2545 adults with severe TBI is characterised in Box 1 and Box 2. Patient admissions were evenly spread across the three BIRP units. Most injuries resulted from motor vehicle accidents; $81 \%$ of patients were male and the mean age at time of injury for the entire sample was 35 years. The median length of admission for rehabilitation was 37 days, and the mean discharge FIM score was 104 . Three-quarters of patients $(74 \%)$ were discharged home, and a third (32\%) required moderate or maximal assistance. The median risk-exposure time was 9.3 years (interquartile range [IQR], 7.4; range, 2.0-19.5). 


\section{Long-term all-cause mortality pattern}

There were 258 deaths recorded at the census date, representing a mortality rate of $10.2 \%$. Death certificates were available from the NSW Registry of Births, Deaths and Marriages or the NDI for 243 deceased patients (94\%). The expected number of deaths in the matched reference population was 81 (3.2\%), yielding an SMR of 3.19 (95\% CI, $2.80-3.60)$. There was a $21 \%$ overrepresentation of male deaths (observed male to female ratio, 9.75:1 compared with a predicted ratio of $8.07: 1)$. The median time from discharge from rehabilitation to death was 4.8 years (range, 1 month to 17.4 years). The difference between the observed discharge after rehabilitation and population-predicted deaths is shown in Box 3A.

The year-by-year SMR for the inception cohort ranged from 12.3 in the first year after discharge $(95 \% \mathrm{CI}$, 3.9-29.3) to 1.25 in the 15th year after discharge (Box 3B). Yearly SMRs were not calculated beyond the 16th year after discharge because there were too few deaths for accurate calculation. Across this interval, the SMR decreased over time with a power law function $\left(y=11.052 x^{-0.7225}, r^{2}=0.843\right)$ potentially enabling prediction of yearly SMR. From the 9th year after discharge, the $95 \%$ CI lower bound intermittently included 1.0, suggesting an elevated mortality for patients after TBI above the general population for at least 8 years after discharge.

\section{Univariate and multivariate risk factors}

The univariate Cox regression analysis identified several risk factors contributing to risk of death (Box 1, Box 2). When entered together into the multivariate Cox regression analysis (Box 4), functional dependence produced the highest hazard ratio, more than triple the mortality risk of an independent subject.

\section{Mortality patterns for specific causes of death}

Cause of death by ICD-10 chapter is provided in Box 5. Excluding neoplasia, the number of deaths in the TBI cohort exceeded those in the statistically derived reference group, with cause-specific SMRs ranging from
2.6 to 14.1. Deaths in the category with the highest SMR, "Symptoms, signs and abnormal clinical and laboratory findings", were due to "natural causes" or were otherwise "not ascertainable".

\section{Discussion}

This multicentre Australian data linkage study followed an inception cohort of 2545 adults with severe TBI over a mean risk-exposure time of 10 years to determine incidence of mortality and associated risks. Mortality among this patient group was 3.2 times greater than that of the general population, a rate at the higher end of the findings of North American studies, ${ }^{9-11,14}$ but consistent with those of the previous Australian study. ${ }^{7}$ Examined year by year after discharge from rehabilitation, the mortality rate was 12 times that predicted for the general population during the first year after discharge and remained higher than the general population for each of the following seven years. This finding provides valuable new information for reviewing assumptions underlying Australian health modelling. ${ }^{6}$

We found that functional dependence at discharge from rehabilitation
3 Predicted versus observed mortality and yearly standardised mortality ratios ( $95 \% \mathrm{Cls}$ ) for 2545 patients with severe traumatic brain injury

A: Number of living participants over the 18 years after their discharge from rehabilitation

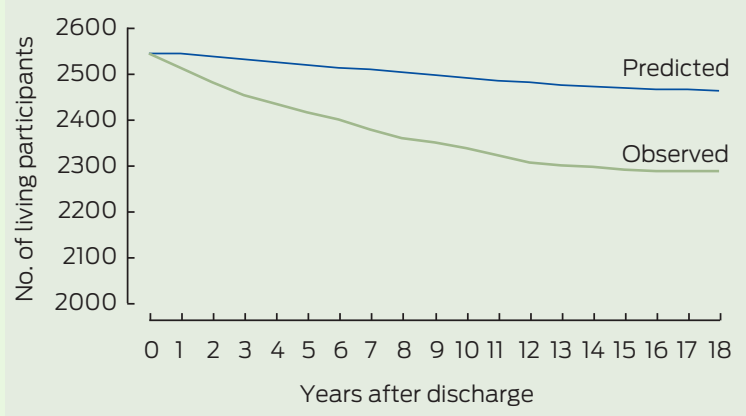

B: Yearly standardised mortality ratios ( $95 \% \mathrm{Cls}$ ) over the 16 years after discharge from rehabilitation

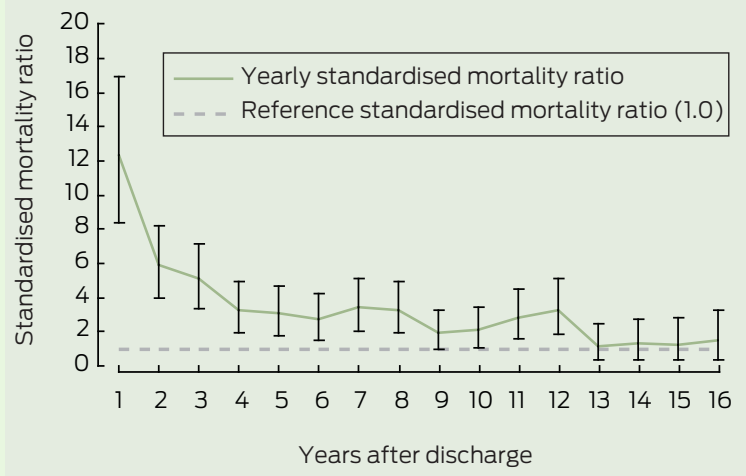

\begin{tabular}{|c|c|c|}
\hline Predictive factors in multivariate analysis & Hazard ratio $(95 \% \mathrm{Cl})$ & $P$ \\
\hline \multicolumn{3}{|l|}{ Sex } \\
\hline Female (reference group for Cox regression) & 1 & \\
\hline Male & $2.24(1.38-3.62)$ & 0.001 \\
\hline \multicolumn{3}{|l|}{ Age } \\
\hline 16-20 years (reference group for Cox regression) & 1 & \\
\hline $21-25$ years & $0.57(0.24-1.33)$ & 0.19 \\
\hline $26-35$ years & $1.13(0.58-2.20)$ & 0.73 \\
\hline $36-45$ years & $2.01(1.07-3.80)$ & 0.03 \\
\hline$\geqslant 46$ years & $3.25(1.80-5.87)$ & $<0.001$ \\
\hline Pre-injury history of epilepsy & $2.11(1.35-3.30)$ & 0.001 \\
\hline Pre-injury history of alcohol/drug misuse & $2.39(1.76-3.25)$ & $<0.001$ \\
\hline Occurrence of aspiration pneumonia & $1.79(1.10-2.91)$ & 0.02 \\
\hline \multicolumn{3}{|l|}{ Discharge destination } \\
\hline Private house (reference group for Cox regression) & 1 & \\
\hline Ongoing rehabilitation/medical care & $0.98(0.65-1.47)$ & 0.91 \\
\hline Care facility (nursing home or hostel) & $1.94(1.28-2.94)$ & 0.002 \\
\hline \multicolumn{3}{|l|}{ Discharge functional independence measure scores } \\
\hline Independent (108-126) (reference group for Cox regression) & 1 & \\
\hline Moderate assistance (55-107) & $1.06(0.72-1.57)$ & 0.76 \\
\hline Maximal assistance (18-54) & $3.39(2.22-5.19)$ & $<0.001$ \\
\hline
\end{tabular}

* Variables removed during backward multivariate Cox regression were length of stay and admission functional independence measure score. 
5 Causes of death based on relevant ICD-10 chapters, number of observed and predicted deaths with cause-specific SMR

ICD-10

Chapter Title

Observed

Certain infectious and parasitic diseases
Neoplasms
Diseases of blood
Endocrine
Mental and behavioural disorders
Diseases of the nervous system
Diseases of the circulatory system
Diseases of the respiratory system
Diseases of the digestive system
Diseases of genitourinary system
Symptoms, signs and abnormal clinical and laboratory findings
External causes of mortality ${ }^{\dagger}$
Cause of death pending
Total

$\begin{array}{rrc}4 & 1.4 & - \\ 29 & 28.8 & 1.0(0.7-1.4) \\ 1 & 0.3 & - \\ 2 & 2.2 & - \\ 9 & 1.7 & 5.4(2.4-9.6) \\ 14 & 2.2 & 6.4(3.4-10.3) \\ 52 & 19.9 & 2.6(1.9-3.4) \\ 49 & 4.8 & 10.2(7.5-13.4) \\ 15 & 2.9 & 5.2(2.9-8.3) \\ 3 & 0.9 & - \\ 8 & 0.6 & 14.1(5.9-25.8) \\ 54 & 10.3 & 5.2(3.9-6.7) \\ 18 & & \\ 258 & & \end{array}$

ICD-10 = International statistical classification of diseases and related health problems, 10th revision. SMR = standardised mortality ratio.

$*$ SMR ( $95 \%$ CI) not calculated for causes of death for which fewer than five deaths were observed, because of the inaccuracy of the prediction. $\dagger$ External causes of mortality include transport accidents, falls, accidental drowning and submersion, other accidental threats to breathing, exposure to smoke, fire and flames, accidental poisoning by and exposure to noxious substances, intentional self-harm, assault and inhalation of gastric contents/food. was the strongest factor associated with an increased risk of death in adults with severe TBI, supporting findings from Australian ${ }^{17}$ and international research. ${ }^{9-13,25}$ The next greatest risk factor associated with increased mortality in the cohort with TBI was older age at injury. Adults aged 36 years and older were two to three times more likely to die during the study period than young adults. These age-related relativities are also observed in the general, non-injured population, $^{23}$ which led to the observed normalisation of year-byyear SMR with increasing years after injury. The interaction between agerelated mortality risk and longitudinal SMR warrants further study.

In the general population, males at all ages have elevated mortality rates compared with females, but the mortality rate of males in this cohort was $20 \%$ higher than would be expected. This finding confirms those of previous studies, ${ }^{1,7,10,15-17}$ and remains unexplained. In our study, all but one of the deaths due to external causes (eg, transport accidents, falls, assault and intentional self-harm) were in males. Similarly, deaths from circulatory disease (including ischaemic heart disease, myocardial infarction and cerebrovascular disease) were almost exclusively observed in males. This observation may go some way to explaining the consistent over-representation of males in studies of late mortality after TBI. In particular, factors affecting external causes of death, such as altered regulation of behaviour or impaired judgement, and circulatory diseases warrant further examination.

Discharge from rehabilitation to aged care facilities doubled the risk of mortality after TBI. This risk factor remained significant even when controlling for level of functional dependency at discharge, suggesting that discharge to an aged care facility contributed a unique mortality risk independent of the level of assistance needed by the person at discharge from rehabilitation as measured by FIM. It is unclear whether this effect was the result of characteristics related to individual patients or services

The inception cohort design, implemented across multiple rehabilitation centres, resulted in a large sample of working-age people undergoing specialty rehabilitation after TBI in NSW, thereby improving the rigour of data beyond those of the single Australian study conducted to date. In contrast to other Australian states, admissions in the NSW BIRP occur systematically and without the funding constraints evident in some international settings. However, our study was limited to primarily working-age adults with severe TBI, and further research is required into patterns of mortality in people with mild TBI, and children, the elderly and indigenous Australians with severe TBI. Findings about cause of death reported in our study may underestimate true rates, because data on cause of death was undetermined for 18 deaths. Finally, further research will be required to determine whether systematic rehabilitation programs offer any advantage in terms of the late mortality of survivors of severe TBI.

Acknowledgements: We gratefully acknowledge the statistical advice provided by Karen Byth of Westmead Hospital, and the financial support provided by the New South Wales Government Lifetime Care and Support Authority to complete this study (research grants 08157 and 10588)

Competing interests: No relevant disclosures.

Received 25 Jan 2011, accepted 29 Jun 2011.

1 Bruns J Jr, Hauser WA. The epidemiology of traumatic brain injury: a review. Epilepsia 2003; 44 Suppl 10: 2-10.

2 Khan F, Baguley IJ, Cameron ID. Rehabilitation after traumatic brain injury. Med J Aust 2003; 178: 290-295.

3 Brown AW, Leibson CL, Malec JF, et al. Long-term survival after traumatic brain injury: a population-based analysis. NeuroRehabilitation 2004; 19: 37-43.

4 Fearnside MR, Cook RJ, McDougall P, McNeil RJ. The Westmead Head Injury Project outcome in severe head injury. A comparative analysis of prehospital, clinical and CT variables. Br J Neurosurg 1993; 7: 267-279.

5 Myburgh JA, Cooper DJ, Finfer SR, et al. Epidemiology and 12-month outcomes from traumatic brain injury in Australia and New Zealand. J Trauma 2008; 64: 854-862.

6 Access Economics. The economic cost of spinal cord injury and traumatic brain injury in Australia. Report by Access Economics for the Victorian Neurotrauma Initiative. Canberra: Access Economics, 2009: 31.

7 Baguley IJ, Slewa-Younan S, Lazarus R, Green A. Long-term mortality trends in patients with traumatic brain injury. Brain Inj 2000; 14: 505-512.

8 Colantonio A, Escobar MD, Chipman M, et al. Predictors of postacute mortality following traumatic brain injury in a seriously injured population. J Trauma 2008; 64: 876-882.

9 Harrison-Felix C, Whiteneck G, DeVivo MJ, et al. Mortality following rehabilitation in the Traumatic Brain Injury Model Systems of Care. NeuroRehabilitation 2004; 19: 45-54.

10 Harrison-Felix CL, Whiteneck GG, Jha A, et al. Mortality over four decades after traumatic brain injury rehabilitation: a retrospective cohort study. Arch Phys Med Rehabil 2009; 90: 1506-1513.

11 Ratcliff G, Colantonio A, Escobar M, et al. Longterm survival following traumatic brain injury. Disabil Rehabil 2005; 27: 305-314.

12 Shavelle R, Strauss D. Comparative mortality of adults with traumatic brain injury in California, 1988-97. J Insur Med 2000; 32: 163-166.

13 Shavelle RM, Strauss D, Whyte J, et al. Long-term causes of death after traumatic brain injury. $A m \mathrm{~J}$ Phys Med Rehabil 2001; 80: 510-516.

14 Ventura T, Harrison-Felix C, Carlson N, et al. Mortality after discharge from acute care hospitalization with traumatic brain injury: a 
population-based study. Arch Phys Med Rehabil 2010; 91: 20-29.

15 Selassie AW, McCarthy ML, Ferguson PL, et al. Risk of posthospitalization mortality among persons with traumatic brain injury, South Carolina 1999-2001. J Head Trauma Rehabil 2005; 20: 257-269.

16 Strauss DJ, Shavelle RM, Anderson TW. Longterm survival of children and adolescents after traumatic brain injury. Arch Phys Med Rehabil 1998; 79: 1095-1100.

17 Baguley IJ, Nott MT, Slewa-Younan S. Long-term mortality trends in functionally-dependent adults following severe traumatic-brain injury. Brain Inj 2008; 22: 919-925.
18 Harrison-Felix C, Whiteneck G, DeVivo MJ, et al. Causes of death following 1 year postinjury among individuals with traumatic brain injury. J Head Trauma Rehabil 2006; 21: 22-33.

19 Harradine PG, Winstanley JB, Tate R, et al. Severe traumatic brain injury in New South Wales: comparable outcomes for rural and urban residents. Med J Aust 2004; 181: 130-134.

20 NSW Health. NSW Brain Injury Rehabilitation Program. http://www.health.nsw.gov.au/ initiatives/birp/index.asp (accessed Nov 2011).

21 Shores EA, Marosszeky JE, Sandanam J, Batchelor J. Preliminary validation of a clinical scale for measuring the duration of post- traumatic amnesia. Med J Aust 1986; 144: 569-572.

22 Uniform Data System for Medical Rehabilitation. Guide for the uniform data set for medical rehabilitation (including the FIM instrument) Buffalo, NY: Uniform Data System for Medical Rehabilitation, 1997.

23 Australian Bureau of Statistics. Deaths, Australia. Canberra: ABS, 2008. (ABS Cat. No. 3302.0.)

24 Vandenbroucke JP. A shortcut method for calculating the 95 per cent confidence interval of the standardized mortality ratio [letter]. Am J Epidemiol 1982; 115: 303-304.

25 Strauss D, Shavelle R, DeVivo MJ, et al. Life expectancy after traumatic brain injury [letter]. NeuroRehabilitation 2004; 19: 257-258. 